\title{
Evaluation of a Microwave Biosensor for On-chip Electroporation and Efficient Molecular Delivery into Mammalian Cells
}

A. Tamra, D. Dubuc, Member, IEEE, M.P. Rols, and K. Grenier, Member, IEEE

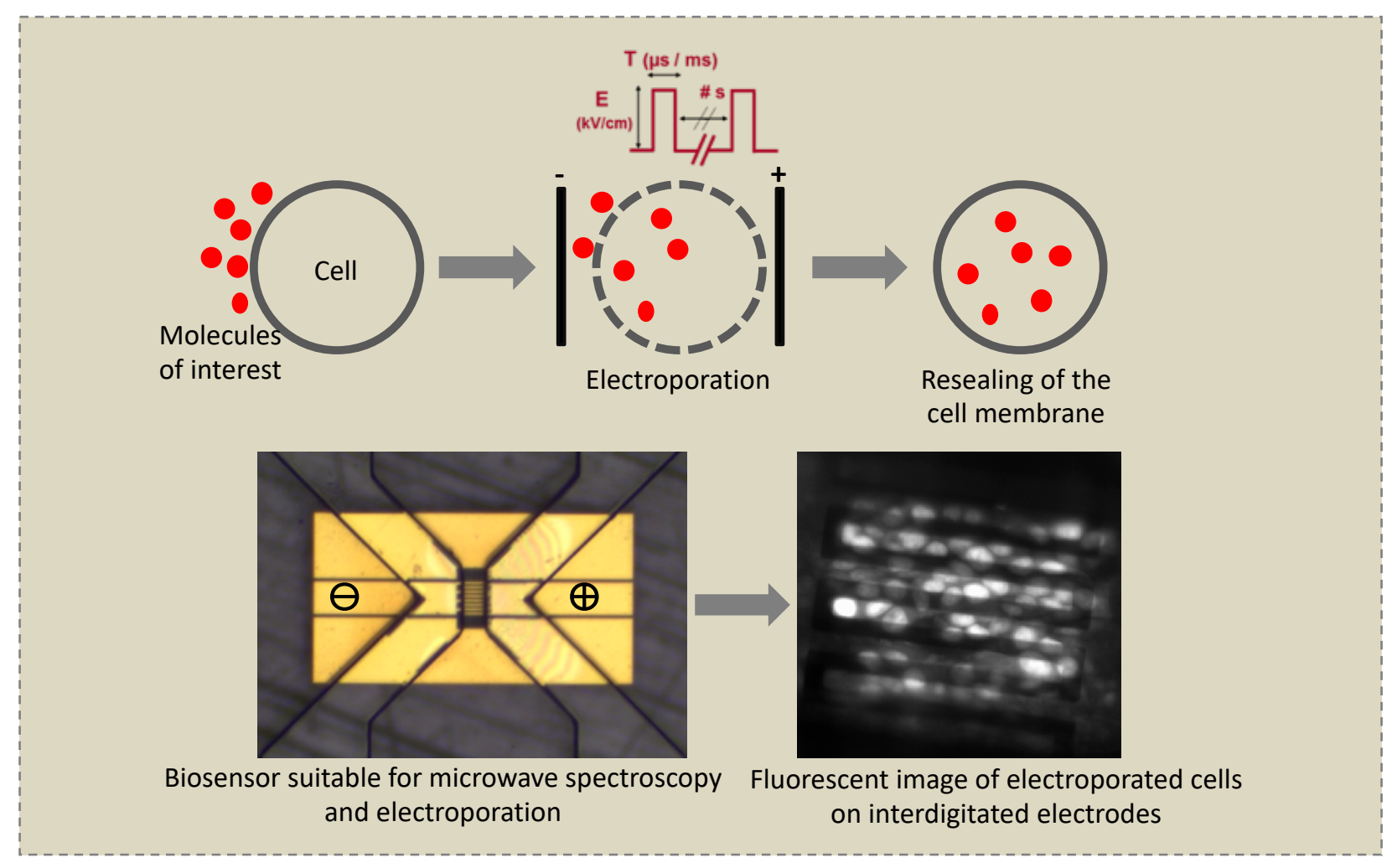

Microwave biosensor suitable for on chip electroporation: delivery of a non-permeant molecule into the cells.

\section{Take-Home Messages}

- Electromagnetic fields are utilized for enhanced drugs delivery into cells with controlled and on-chip electroporation.

- High efficient molecular delivery into mammalian cells is demonstrated with a microdevice able to provide both controlled cell electroporation and microwave sensing for cellular characterization.

- The evaluation of drugs, genes or proteins into cells for therapeutic applications is targeted.

- On-chip electroporation enables high molecular delivery into cells, while maintaining a high viability rate and therefore overcoming some of the drawbacks of conventional electroporation systems. 


\title{
Evaluation of a Microwave Biosensor for On-chip Electroporation and Efficient Molecular Delivery into Mammalian Cells
}

\author{
A. Tamra, D. Dubuc, Member, IEEE, M.P. Rols, and K. Grenier, Member, IEEE
}

\begin{abstract}
In this study, a microwave biosensor based on interdigitated electrode layout is evaluated as an on-chip electroporation system applicable to electroporation of adherent cell monolayers. The present device is designed in a manner that allows electrical microwave spectroscopy measurements in subsequent studies. We demonstrate the applicability of our device for electroporation-mediated molecule transfer of adherent cells in standard laboratory conditions. Application of electric pulses (6 $\mathrm{V}, 100 \mu \mathrm{s}, 1 \mathrm{~Hz})$ to the cells induces successful delivery of a fluorescent probe $(\sim 98 \%)$, while maintaining a high viability rate $(\sim 100 \%)$. The comparable delivery rate to existing systems along with the improved viability suggest that our proposed electroporation system has great potential in the research fields, considering that our system is conceived to perform electrical microwave spectroscopy measurements after electroporation.
\end{abstract}

\section{Keywords — Electroporation, On-chip, Microdevice, Dielectric Spectroscopy.}

\section{INTRODUCTION}

$\mathrm{I}_{\mathrm{p} \text { r }}^{\mathrm{N}}$ NTRODUCING molecules of interest, such as genes, proteins or drugs, into living cells might have a considerable therapeutic impact. However, the plasma membrane acts as a physical barrier that prevents the entry of exogenous molecules inside the cell. In order to overcome this barrier, numerous methods have been developed including viral vectors, chemical treatments and physical approaches, etc. One of the physical approaches elaborated in the 1970s, by Neumann E. and K. Rosenheck [1] consists in applying well-controlled electric pulses (in amplitude and duration) [2] in order to transiently permeabilize the plasma membrane of cells. This method allows the entry and exit of countless types of molecules of interest [1] without the use of cell-damaging reagents or viruses. The first studies on electroporation reported permanent damages caused to the cellular membrane, which induced cell death: irreversible electroporation took place [3]. This type of electroporation might be used as a nonthermal tumor ablation treatment, as well as a method for eradication of microorganisms in the industrial field. On the other hand, it has been showed that the permeabilization of the cell membrane can indeed be a reversible procedure: the

This work was supported by the Toulouse COMUE and the French Midi-Pyrenees region. This work was also partly supported by LAASCNRS micro and nano technologies platform, member of the French RENATECH network.

A. Tamra, D. Dubuc and K. Grenier are with the National Scientific Research Center, Laboratory of Analysis and Architecture of Systems, 31400 Toulouse, France (phone: +33 56133 6291; fax: +33 56133 6969; emails: atamra@laas.fr; dubuc@laas.fr; grenier@laas.fr).

A. Tamra and M-P. Rols are with the Institute of Pharmacology and Structural Biology, 31077, Toulouse, France (phone: +33 56117 5900; fax: +33 56117 5994; e-mails: tamra@ipbs.fr; rols@ipbs.fr). cell is able to recover after the application of the electric pulses [4]. The latter is used to deliver drugs (electrochemotherapy), DNA (electrogenotherapy) or proteins into the cells and its mechanisms remain poorly understood. Electroporation enables an important number of applications in various domains, and some of them include the insertion of proteins into cell membrane, cell fusion, eradication of bacteria, introduction of small and large molecules into the cell, as well as tumour ablation [5].

In a conventional electroporation process, wellcontrolled electric pulses are delivered to cells placed in a cuvette equipped with embedded electrodes [6]. A pulse generator capable of generating high voltage pulses is also required. These conventional systems are widely used; despite some disadvantages such as the high voltage input required resulting in the use of relatively high cost pulse generators, excess heat generation, metal ion dissolution and local $\mathrm{pH}$ variation, etc.

The microfluidic-based on-chip electroporation is receiving global attention and is rapidly growing due to its ability to overcome many drawbacks of the bench-scale electroporators [7], [8]. First, on chip electroporation allows to reduce the distance between the electrodes and decrease the potential differences. Few volts are sufficient to give high electric field strengths [9] and both millisecond and nanosecond pulses may be applied. An additional advantage of an on-chip microscale system would reduce the relatively high cost of high voltage pulse generators. Furthermore, the potential risk of overheating can be minimized thanks to the miniaturization, as heat dissipates more effectively and quickly within the micro-device due to the high surface to volume ratio. Second, these miniaturized systems allow the use of small amounts of samples and a reduced consumption of expensive reagents. Third, the transparent 
materials used in the fabrication of these systems (such as polydimethylsiloxane PDMS, SU-8 and glass) allow microscopic in-situ surveillance, and real-time monitoring of electroporation process using fluorescent markers, promoting better understanding of the mechanism [9]. Finally, these systems can also incorporate multiple functionalities such as dielectrophoresis DEP [10], electrorotation [11], impedance spectroscopy [12], microwave dielectric spectroscopy [13], etc. for subsequent analysis.

The current research is focused on microfluidic electroporation. Over the last twenty years, it has shown important advances in this field. A wide choice of cell types and applications have been reported. Various systems were developed for adherent cells [14], [15], [16], [17], [18], suspension cells [19], [20], [21], [22] and even single cells [23], [24], [25]. Thanks to these microdevices, the determination of the optimal electrical parameters in terms of electric field intensity and pulse duration is easier and faster. On the other hand, the developed systems can be used for different types of applications. For example, many studies have reported the use of microfluidic systems in order to perform cell lysis after the application of irreversible electroporation [26], [27], [28]. Moreover, the electrically mediated gene transfection is receiving great attention, further motivating the researches working on its miniaturization. Many examples exist in the literature illustrating the on-chip electro-transfection [29], [30], [31]. Finally, the field of Electro Chemo Therapy (ECT), combining cytotoxic agents to an electric field, is rapidly arising in the domain of cancer treatment [32], [33]. A good evaluation of the cell type, the used drug and the electric field parameters need to be established prior any kind of treatment. For this purpose, some researchers are working on the development of new microsystems adapted to the study of ECT [34], [35].

Routinely used methods of assessment of the effectiveness of in-vitro electroporation are already effective [36], [37]. Nevertheless, they require the use of molecular reporters and imaging techniques, which can be time-consuming and abusive for the cells. On the other hand, electrical methods are already used to evaluate the effect of electroporation on the membrane integrity of the treated cells or tissues. When electroporation is applied to the cells, membrane permeabilization occurring over a short period of time results in a modification of the overall dielectric properties of the cell. On larger time scales, the internal properties of the cells are altered due to ion transfer through the membrane [38]. Consequently, the monitoring of the dielectric properties by spectroscopy of cells after electroporation can be used as a detection technique allowing the study of the impact of the electric field-cell interaction and the resulting physiological change [39]. Herein lays the interest of our microelectrode assembly presented in this work, since it integrates on-chip electroporation and can perform microwave dielectric spectroscopy monitoring of cells subjected to in situ electroporation.

In the present paper, we report our work on the on-chip electroporation performed using the microfluidic chip developed by Chen et al. [40] for microwave dielectric spectroscopy of cells. The micro-device's architecture combining microfluidic and electrical functionalities is described. After a step of experimenting on the macrometric scale (conventional electroporation), the success of on-chip electroporation is shown, while preserving a high cellular viability rate. Our proposed microdevice is suitable for subsequent microwave spectroscopy measurements of cells under electroporation.

\section{MAterials AND MethodS}

\section{A. Cells}

HCT116 cells (ATCC \# CCL-247) are adherent epithelial human cells having a high proliferative capacity, derived from a colorectal cancer. They are grown in "Dulbecco's Modified Eagle Medium" (DMEM) (Gibco-Invitrogen, Carlsbad, CA, USA) containing $4.5 \mathrm{~g} / \mathrm{L}$ glucose, Lglutamine and pyruvate. This medium is supplemented with $10 \%$ foetal calf serum FCS and a mixture of antibiotics 100 $\mathrm{U} / \mathrm{ml}$ penicillin and $100 \mathrm{ug} / \mathrm{ml}$ streptomycin. The cells are incubated at $37^{\circ} \mathrm{C}$ under $5 \% \mathrm{CO}_{2}$.

\section{B. Microchip Architecture}

The microdevice used for this work is designed and developed by Chen et al. [40] and fabricated in the clean room, using the well-established microfabrication processes (deposition, photolithography and etching of films notably). During the fabrication process, biocompatibility and transparency criteria are respected.

Since the microdevice is first a microwave sensor for dielectric spectroscopy of cells, it includes mandatory microwave electrodes specifications. The microdevice comprises a coplanar waveguide, i.e. two ground planes placed apart a central conductor, with an interdigitated capacitor within the central conductor, in the middle of the transmission line. The coplanar waveguide presents a central width of $150 \mu \mathrm{m}$ and slots of $15 \mu \mathrm{m}$ wide to exhibit a $50 \Omega$ characteristic impedance and is compatible with standard $150 \mu \mathrm{m}$ microwave probes. The interdigitated electrodes are $10 \mu \mathrm{m}$ wide, with slots of $10 \mu \mathrm{m}$ large and $300 \mathrm{~nm}$ thick. The sensing area is a square of 150 by 150 $\mu \mathrm{m} 2$. These gold electrodes are patterned onto a quartz substrate by using lift-off technique. For on-chip electroporation, electric field is applied between the input and output of the central conductors of the sensor.

SU-8 walls are patterned on the quartz substrate above the electrodes in order to create the microfluidic channel. This channel is $1 \mathrm{~mm}$ wide with a restricted sensing zone of $150 \mu \mathrm{m}$, and $250 \mu \mathrm{m}$ high. Figure 1 shows the microdevice (a) with the interdigitated electrodes (b), between which electric field pulses are applied. The fabrication of the microdevice is described more extensively elsewhere [40].

During their culture into the microdevice (Figure 1. c), 
the cells adhere not only on the interdigitated electrodes (zone where the electric field is generated) but also along the microfluidic channel (zone without an electric field). These cells thus form our internal negative control.
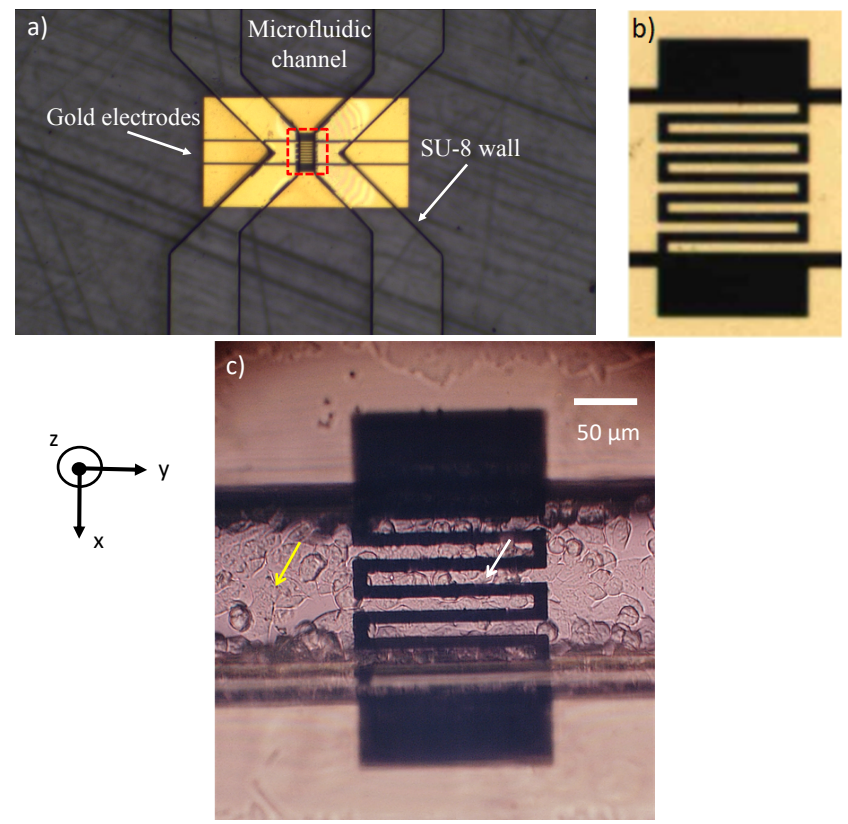

Fig. 1. (a) Microfluidic chip for electroporation. The microchip presents golden electrodes patterned on a quartz substrate topped with a SU-8 fluidic channel. (b) Zoom on the interdigitated electrodes. The electrodes are connected to an external function generator. (c) HCT116 cells cultured inside the microfluidic channel. White arrow indicates cells on the electrodes and yellow arrow indicate cells in the microfluidic channel (internal negative control).

\section{Electric Field Simulation}

In order to understand the microelectroporation process, electric field distribution is evaluated by a finite element method (FEM) software (COMSOL Multiphysics ${ }^{\circledR}$ ). A 2D model (in X-Z plane, see definition in Fig. 1) of the microdevice cross section is presented in Figure 2, assuming the field intensity constant along (y) axis due to the subwavelength of the interdigitated electrodes. Our device has four pairs of interdigitated electrodes, but in this simulation, we only study the electric field developed by one periodic pattern, which is constituted by two half electrodes $(0 \mathrm{~V})$ and a central one $(5 \mathrm{~V})$. The result is transposable to each electrode, except for the fingers located at the edges, for which the simulation overpredicts the field intensity. The model consists of electrodes on quartz substrate surrounded with electroporation medium. The relative permittivity and conductivity of the medium are set to 77.4 and $1.7 \mathrm{~S} / \mathrm{m}$, respectively. The potential difference between the positive and the negative electrode is set to 5 Volts. The simulation shows that the distribution of the electric field is quasi uniform in (x) direction and and non-uniform in the $(\mathrm{z})$ one. The electroporation will therefore be independent of the position of the cells over the electrodes (x-y plane). Moreover, it also shows that with a potential difference as low as 5 Volts, an important electric field is produced at the vicinity of the electrodes (few micrometers) up to $3 \mathrm{kV} / \mathrm{cm}$. The field intensity significantly decreases as the distance from the electrodes rises: at 5,10 and $15 \mathrm{um}$, the field strength decreases down to $1.9,0.7$ and $0.2 \mathrm{kV} / \mathrm{cm}$ respectively. These former values state the range of the effective field intensity submitted to the cells. Nevertheless, such a simulation is not accurate enough to predict the relationship between the effective electric field intensity and the applied voltage to the device (range is only predicted). Cells are indeed not considered, whereas their presence significantly affects the electrical field. This relationship will be experimentally evaluated in section III.C.

\section{Electric Field Generation Systems}

During our experiments, we use two different types of electric field generators, dedicated either to macroscale or to microscale electroporation. For the macrometric scale electroporation (or conventional electroporation), that is usually used in laboratory and clinical experiments, electric pulses are delivered with a high voltage pulse generator (ELECTRO cell S20) from Betatech (Bordeaux, France), using two stainless steel parallel plate electrodes $(10 \mathrm{~mm}$ length, $0.5 \mathrm{~mm}$ thick, and $4 \mathrm{~mm}$ inter-electrode distance). The pulses are displayed on an LCD screen using integrated pulse control software. As for the micrometric scale electroporation, which is associated with our microdevice and suitable for the on-chip electroporation, we use the Tektronix AFG3022C pulse generator, controlled by a Tektronix TDS 210 oscilloscope to monitor the pulses. The generator delivers the electrical pulses to the cells by means of needle electrodes placed in contact with the central conductor of the coplanar transmission lines of the microdevice.

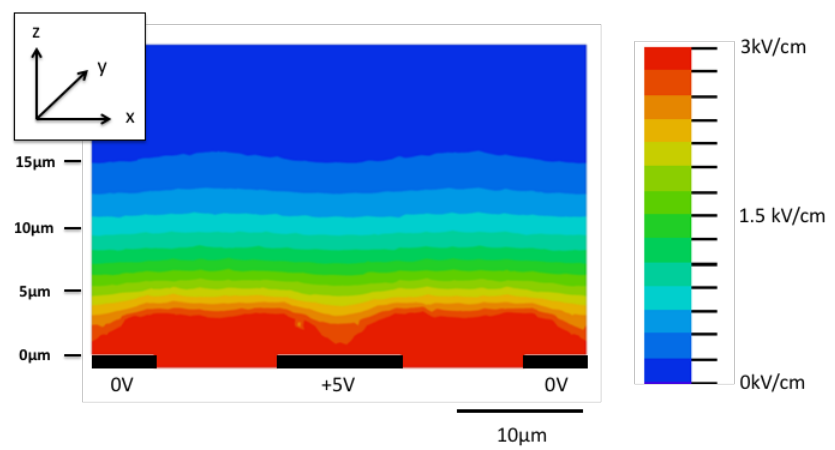

Fig. 2. Finite element simulation showing the distribution of the electric field in the channel with interdigitated electrodes. The modulus of the electric field $(\mathrm{V} / \mathrm{m})$ is shown for $5 \mathrm{~V}$ of potential difference between the positive and negative electrodes. The system is periodic and only one pattern is shown.

\section{E. Experimental Procedure}

Before proceeding with electroporation, cells are cultured for 24 hours in petri dishes (conventional electroporation) or on the device (micrometric electroporation). In this work, we are interested in the clinical applications of electroporation, thus we studied these parameters: 8 pulses at $0.2-1.4 \mathrm{kV} / \mathrm{cm}$ of amplitude with $100 \mu$ s pulse duration, at a frequency of $1 \mathrm{~Hz}$. In order to estimate the permeabilization rate after electroporation, we add 
Propidium Iodide PI $(100 \mu \mathrm{M})$ diluted in the pulsation buffer ZAP $\left(10 \mathrm{mM} \mathrm{K} \mathrm{HPO}_{4} / \mathrm{KH}_{2} \mathrm{PO}_{4}, 1 \mathrm{mM} \mathrm{MgCl} 2,250\right.$ $\mathrm{mM}$ of sucrose, $\mathrm{pH}$ 7.4) to the cells. PI is a cell impermeable fluorescent DNA intercalating agent that only penetrates in cells that have lost their membrane integrity. Phase contrast and fluorescence micrographs are taken in each experiment in order to determine the percentage of permeabilized cells. The images are taken with a DMIRB fluorescence microscope equipped with different objectives and a cool snap FxHQ2 camera. In order to estimate the cells viability rates after electroporation, we proceed, 24 hours post electroporation, to the technique of adherent cell counting: the dead cells are considered detached from the petri dish or the microdevice. Several acquisitions in phase contrast are acquired in the pulsation zone using the Leica DMRIB microscope. The cell viability is then measured by counting the number of cells per plane and compared to the condition where the cells have not been treated (negative control). For both permeability and viability tests, the experiments are performed in triplicates.

\section{RESUltS AND Discussion}

\section{A. Conventional electroporation}

Prior the optimization of the use of our microdevice for "on-chip" electroporation, we explored the potential effects of EP on the internalization of exogenous molecules (Propidium iodide) after "conventional" electroporation and the influence of the electric pulses on the viability of the cells. The micrographs in Figure 3 (a) show different groups of cells treated with different intensities of electric field $(0 \mathrm{kV} / \mathrm{cm}$ for the negative control, $0.6,1$ and 1.4 $\mathrm{kV} / \mathrm{cm}$ ) in the presence of PI molecule.

The fluorescing cells present in the negative control photo represent the basal level of cell death of this cell line. The graph shows the evolution of the number of permeabilized cells as function of the intensity of the electric field. The other parameters (number, duration and frequency of the pulses) are kept constant. The internalization of PI is significantly detected when a field of $0.2 \mathrm{kV} / \mathrm{cm}$ is applied and reaches its maximal value around $0.8 \mathrm{kV} / \mathrm{cm}$.

A good compromise should be done when choosing the right parameters in a way that we have a high permeabilization rate along with preserving a high viability rate. For this purpose, cell counting viability assays are conducted 24 hours after electroporation. Compared to the untreated cells (negative control), a decrease in cell density is observed when increasing the intensity of the electroporation field (Figure 4). A quantitative confirmation is established: the cell density in each EP condition is compared with that of the negative control. This allows us to have the percentage of viability as a function of the intensity of the electric field.

Thus, the choice of the suitable electric parameters is done based on the desired subsequent application. In order to have reversible electroporation, adapted for biomedical applications, we choose to apply $0.8 \mathrm{kV} / \mathrm{cm}$ to HCT116 cells, inducing a high permeabilization $(\sim 90 \%)$ and preserving a high viability $(\sim 60 \%)$.

a)
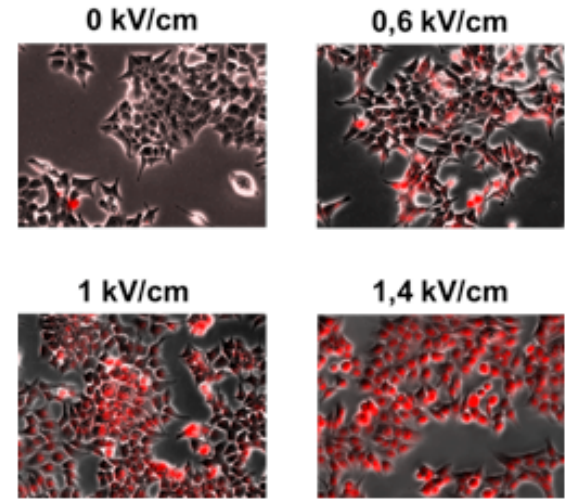

b)

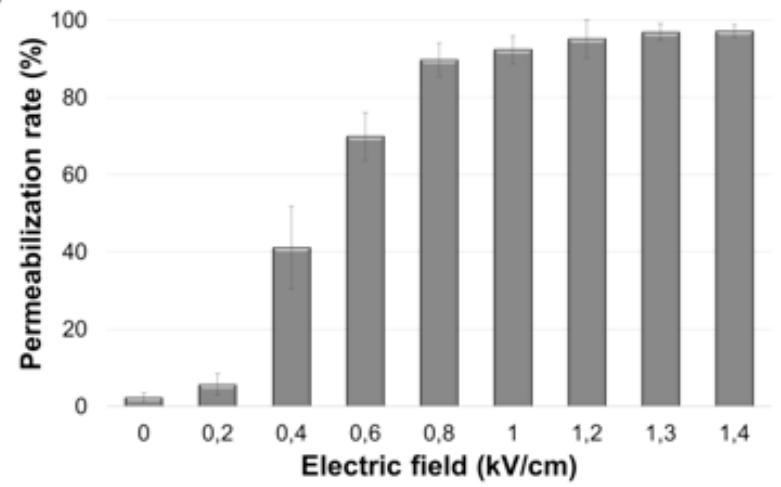

Fig. 3. Evaluation of HCT116 permeability after EP by Propidium Iodide intake. Photos of HCT116 cells after application of 0 (negative control), $0.6,1$ and $1.4 \mathrm{kV} / \mathrm{cm}$. The graph shows the percentage of permeabilized cells after EP $(n=3)$.

\section{B. On-chip electroporation - Qualitative analysis}

In the next section of the work, we show the study that we carried out in order to transpose the conventional macrometric electroporation on a micrometric device with an electroporation zone that does not exceed $150 \mu \mathrm{m}$ and an inter-electrode distance of $10 \mu \mathrm{m}$.

The next step was to validate the process of miniaturization of the electroporation setting. As explained in the materials and methods section, HCT116 cells are cultured 24 hours prior to electroporation in the microdevice. The cells adhere and cover not only the electroporating electrodes, but also the whole surface of the microfluidic channel, which makes it a reliable internal negative control. Figure 5 represents fluorescence images of HCT116 cells cultured in the same microdevice after applying different increased values of voltage (for qualitative analysis, cumulative EP is considered for efficient EP level identification). As shown in figure 5, cells located outside the interdigitated area in the microfluidic channel are not subjected to the electric field and remain intact throughout the experiment. On the other hand, HCT116 cells that are exposed to the electric pulses intake the non-permeant PI dye indicating their permeabilization. The fluorescence emitted by the PI becomes detectable after applying 3 Volts, and a coherent behavior is obtained: an 
increase in the intensity of the electrical pulses induces an increase in the number of permeabilized cells, which reaches $98 \%$ after the application of 6 volts as applied voltage.

a)
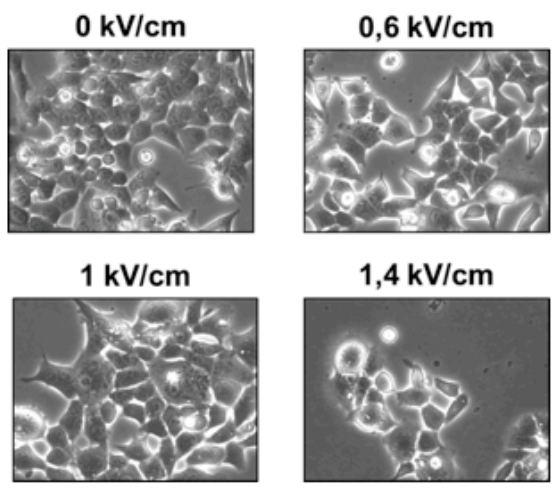

b)

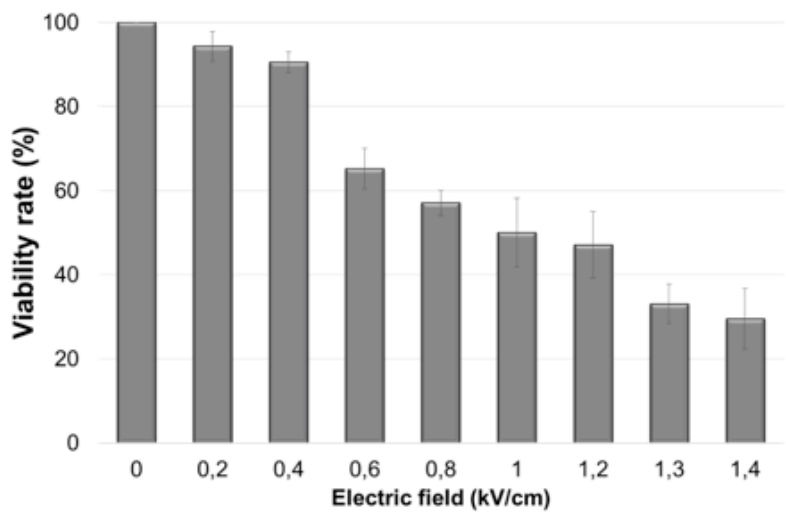

Fig. 4. Evaluation of HCT116 viability after EP. Photos of HCT116 cells after application of 0 (negative control), $0.6,1$ and $1.4 \mathrm{k} / \mathrm{cm}$. The graph shows the percentage of viable cells after EP $(n=3)$.
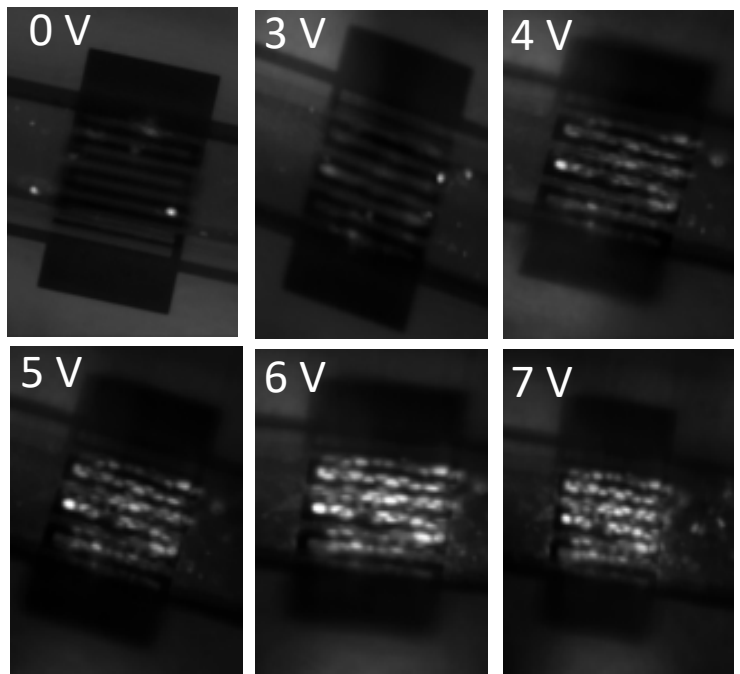

Fig. 5. Fluorescent images of HCT116 cells in the same microdevice after electroporation. Images at different increased values of voltages ( 8 pulses of $100 \mu \mathrm{s}$ at $1 \mathrm{~Hz}$ ). Cells are pulsed in the pulsation buffer with the presence of $100 \mu \mathrm{M}$ of Propidium Iodide PI.

Using the ImageJ software (NIH, Bethesda, USA), an "Interactive 3D surface plot" is obtained from the fluorescence images. It allows illustrating a surface distribution of the intensity of pixels of the corresponding original images. Figure 6 shows two examples: (a) and (c) non-pulsed cells ( 0 Volts applied voltage) and (b) and (d) pulsed cells (6 Volts as applied voltage).
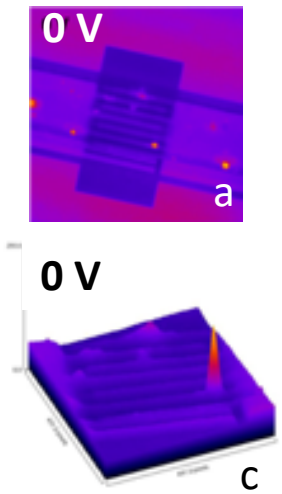
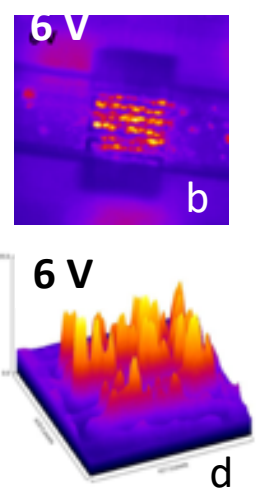

Fig. 6. Interactive 3D surface plot obtained from fluorescence images of the cells in the presence of PI on the microdevice : visualization of the distribution of permeabilized cells and the fluorescence intensity of (a), (c) negative control cells $(0 \mathrm{~V})$ and $(\mathrm{b})$, (d) cell treated with EP (6 V as applied voltage).

At first glance, one can conclude that only the cells that adhere to the electrodes are the ones that are permeabilized by the electric field. By looking a bit closer, we can notice several fluorescent cells that overlap both the electrodes and the space between the electrodes, like shown in Figure 7 (a). In fact, the impression one may have is due to the optical effect of the gold layer. It shows a better reflection of the fluorescence than the quartz substrate Figure 7 (b).

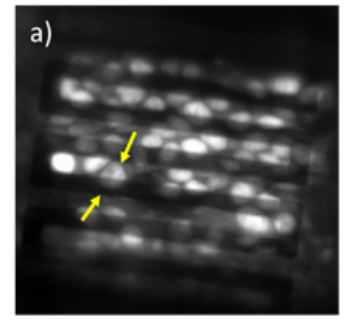

\section{b)}

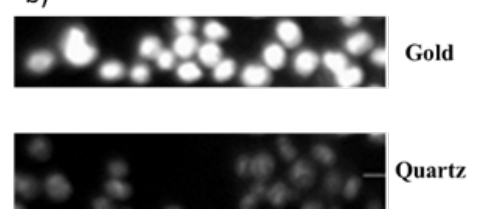

Fig. 7. (a) Zoom on the cells subjected to electroporation at 6 Volts applied voltage, with yellow arrows showing overlapping cell. (b) Comparison of fluorescent intensities on gold and on quartz substrate.

\section{On-chip electroporation - Quantitative analysis}

A quantitative analysis of on-chip electroporation is performed using exactly the same protocol as for conventional electroporation (non-cumulative EP). The study of the fluorescence images allows the quantification of the percentage of permeabilized cells in function of the applied electrical voltage, as well as the estimation of the fluorescence intensity associated with each condition (Figure 8). The increase in the value of the voltage is accompanied by an increase in the number of permeabilized cells and the amount of internalized PI. For example, when applying 4 Volts, a high permeabilization rate $(70 \%)$ is accompanied with a low fluorescence intensity (20 arbitrary unit- a.u.), indicating that a large number of cells are permeabilized but a reduced amount of PI molecules is internalized. At 5 Volts, maximum permeabilization is achieved but the number of internalized molecules does not exceed 30 a.u. It is only at 6 Volts where a permeabilization 
of all the cells is observed with maximum internalization of PI molecules. When pulses of 7 Volts are applied, a maximum permeabilization rate is maintained but accompanied with a decrease in the fluorescence intensity (decrease in the number of PI molecules inside the cell). This can be explained by an irreversible permeabilization of the cells, allowing the entry and exit of the PI molecules.

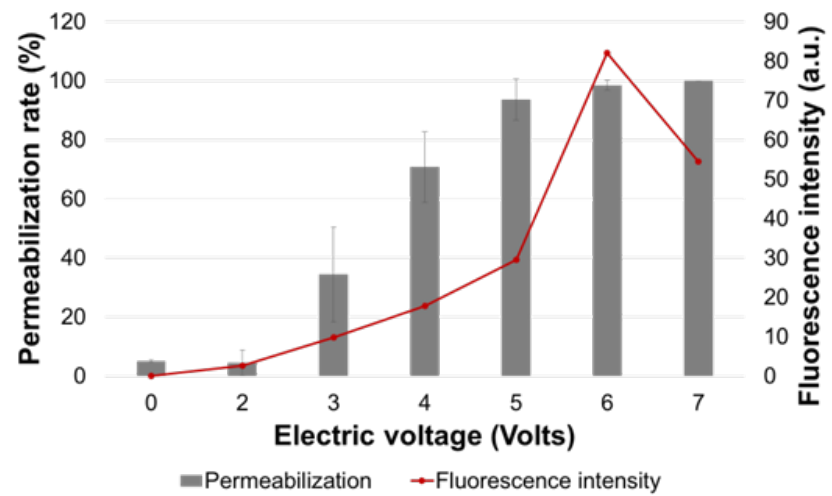

Fig. 8. Quantification of the efficiency of delivery of PI dye and the fluorescence intensity in the HCT116 cells, after the application of 8 pulses at different electric voltages ( $0-7$ Volts), $100 \mu \mathrm{s}, 1 \mathrm{~Hz}(\mathrm{n}=3)$.

By comparing the results of the cellular permeabilization after on-chip electroporation (Figure 8) to those obtained after conventional electroporation (Figure 3), we note the similar shape of both graphs: the permeability tends to increase when increasing the intensity of the electric pulses. Therefore, our microdevice is validated as convenient and compatible for on-chip electroporation functionalities. Moreover, for similar viability rate, we extract a 0.16 $\mathrm{kV} / \mathrm{cm}$ per applied Volt correlation factor between the electric field (in $\mathrm{kV} / \mathrm{cm}$ ) for the conventional electroporation with the applied voltage (in Volt) for the on-chip electroporation. For example, 5 Volts of applied voltage in the case of on-chip electroporation corresponds to an effective electric field intensity of $0.8 \mathrm{kV} / \mathrm{cm}$.

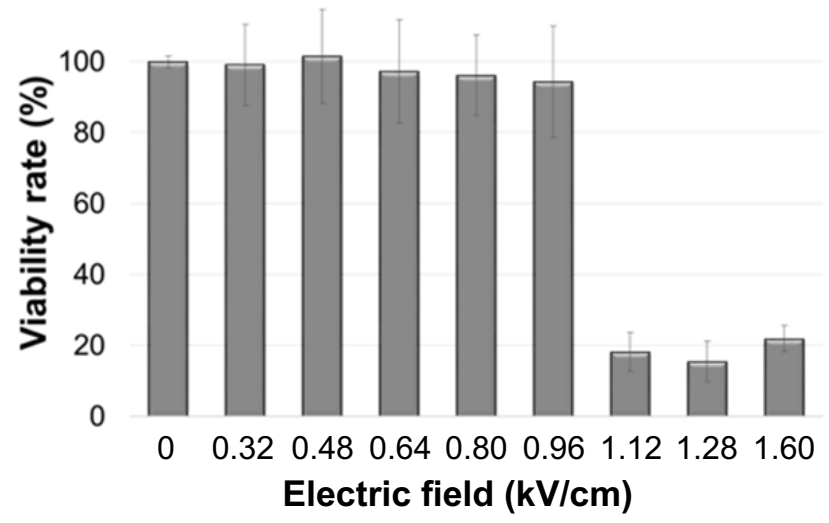

Fig. 9. Viability of HCT116 cells, 24 hours after on-chip electroporation, with different values of electric field $(0-1.6 \mathrm{kV} / \mathrm{cm})(\mathrm{n}=3)$.

The most important requirement in the development of new tools for electroporation is the preservation of a high level of viability of the cells. In order to evaluate the effect of the electrical conditions used on the viability of HCT116 within our microdevice, we have studied the proliferative capacity of the cells, 24 hours after electroporation, by counting the number of cells in each condition and comparing it to the non-electroporated cells (negative control). We therefore consider that a present and adherent cell in our microdevice is a cell that has preserved its viability properties. Figure 9 shows the percentage of viable cells after application of 8 pulses at different electric field conditions $(0-1.6 \mathrm{kV} / \mathrm{cm})$. Viability seems to be preserved no matter what electric field is used up to $0.96 \mathrm{kV} / \mathrm{cm}$, as visible in Figure 10.

Beyond this value, the cells are no longer present on the electrodes, whereas in the juxtaposed regions (for which no field has been established), the cells continue to proliferate normally, as shown in Figure 10. This result is in agreement with the results obtained in the study of permeabilization in the presence of PI: from $1.12 \mathrm{kV} / \mathrm{cm}$, the cells are irreversibly electroporated and therefore, are no longer present on the electrodes, 24 hours after EP. The effect on the cells viability in the microdevice appears to be sharp. Despite the high rate of permeabilization that we obtain when applying $0.96 \mathrm{kV} / \mathrm{cm}$ for example, this value of electric field does not seem to have an effect threatening cellular integrity. On the other hand, once this threshold is exceeded, the EP induces extensive cell death.

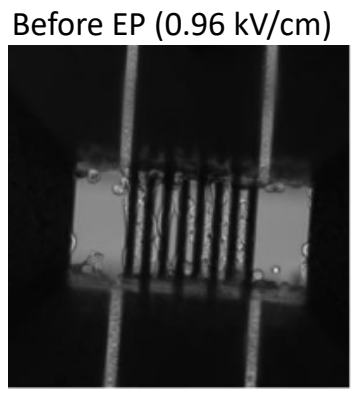

Before EP $(1.12 \mathrm{kV} / \mathrm{cm})$

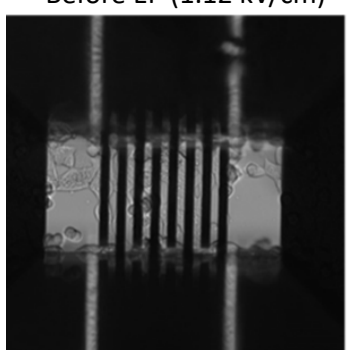

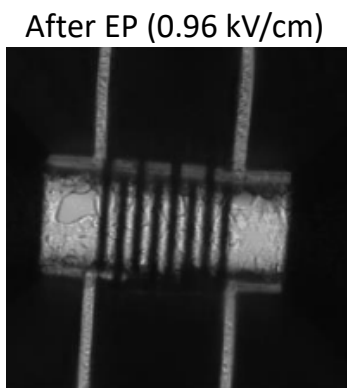

After EP $(1.12 \mathrm{kV} / \mathrm{cm})$

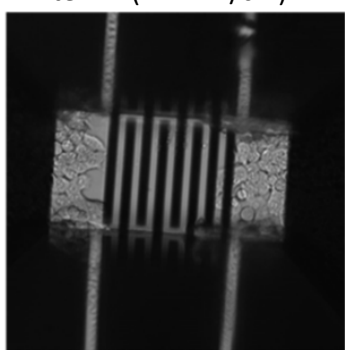

Fig. 10. Photos of HCT116 cells in the microdevice, before and 24 hours after applications of $0.96 \mathrm{kV} / \mathrm{cm}$ and $1.12 \mathrm{kV} / \mathrm{cm}$.

At the end of this study, a high level of viability is preserved after the application of electric field. At 0.96 $\mathrm{kV} / \mathrm{cm}, 94 \%$ of the cells remain present, 24 hours after the electrical treatment on the electrodes. This validates the ability of our microsystem to be used for the evaluation of optimal electroporation conditions. We hypothesize that the high viability rate obtained with the on-chip electroporation and the sharp threshold in Figure 9 are due to the implementation of a better dissipation of energy in the microdevices, minimizing the damage caused by the Joule effect [41], [42], a phenomenon more present with the conventional electroporation. 


\section{CONCLUSION}

In the present study, we present a microdevice with interdigitated electrodes, dedicated to on-chip electroporation and conceived to perform electrical microwave spectroscopy measurements in subsequent studies. A first step of studying the HCT116 cell line subjected to conventional electroporation is carried out, in order to better characterize and understand its response to the electric field. Then, the distribution of the electric field within our microdevice is verified, using the finite element simulation. These simulations show a non-homogeneous distribution of the electric field, but also that an intense electric field level is reached for voltages of the order of a few applied volts. Afterwards, experiments of on-chip electroporation are launched and show that, with our system, a high rate of cellular permeabilization ( $\sim 98 \%)$ accompanied by a preservation of the integrity of the cellular membrane $(\sim 100 \%)$, up to $0.96 \mathrm{kV} / \mathrm{cm}$ may be obtained. The importance of the successful on-chip electroporation lies in the fact that it allows us to overcome some of the drawbacks of conventional electroporation. In fact, the reduced distance between the electrodes allows the use of low voltages, which can be obtained with conventional and low-cost generators. Furthermore, the micrometric size of the device allows the use of small volumes of products and reagents and thus contributes to the increase of the analysis efficiency as well as its possible parallelization. More importantly, high viability rates are obtained when operating in microdevices, compared to the conventional macrometric electroporation. Nevertheless, the most important interest of our system remains in its ability to perform, in future studies, electroporation associated with microwave dielectric spectroscopy measurements. This would contribute to a better understanding of the effect of electroporation on living cells, as performed in the $\mathrm{kHz}-\mathrm{MHz}$ range in [43].

\section{ACKNOWLEDGMENT}

The authors would like to thank Jelena Kolosnjaj for her constructive criticism of the manuscript.

\section{REFERENCES}

[1] E. Neumann et K. Rosenheck, «Permeability changes induced by electric impulses in vesicular membranes », J. Membr. Biol., vol. 10, $\mathrm{n}^{\mathrm{o}} 1,1972$.

[2] J. Teissie, M. Golzio, et M. P. Rols, « Mechanisms of cell membrane electropermeabilization: a minireview of our present (lack of?) knowledge », Biochim. Biophys. Acta, vol. 1724, n 3, pp. 270-280, août 2005 .

[3] B. Rubinsky, G. Onik, et P. Mikus, «Irreversible electroporation: a new ablation modality--clinical implications », Technol. Cancer Res. Treat., vol. 6, n ${ }^{\circ}$ 1, pp. 37-48, Feb. 2007.

[4] T. Kotnik, P. Kramar, G. Pucihar, D. Miklavcic, et M. Tarek, « Cell membrane electroporation- Part 1: The phenomenon », IEEE Electr. Insul. Mag., vol. 28, nº 5, pp. 14-23, Sept. 2012.

[5] T. Kotnik, W. Frey, M. Sack, S. Haberl Meglič, M. Peterka, et D. Miklavčič, "Electroporation-based applications in biotechnology ", Trends Biotechnol., vol. 33, $\mathrm{n}^{\circ}$ 8, pp. 480-488, Aug. 2015.

[6] H. Potter, "Electroporation in biology: methods, applications, and instrumentation », Anal. Biochem., vol. 174, n 2, pp. 361-373, Nov.
1988.

[7] M. B. Fox et al., «Electroporation of cells in microfluidic devices: a review ", Anal. Bioanal. Chem., vol. 385, n 3, pp. 474-485, June 2006.

[8] S. Kemmerling et al., « Single-cell lysis for visual analysis by electron microscopy », J. Struct. Biol., vol. 183, n 3, pp. 467-473, Sept. 2013.

[9] T. Geng et C. Lu, « Microfluidic Electroporation for Cellular Analysis and Delivery », Lab. Chip, vol. 13, n 19, pp. 3803-3821, Oct. 2013.

[10]E. Salimi et al., « Electroporation and dielectrophoresis of single cells using a microfluidic system employing a microwave interferometric sensor ", in 2013 IEEE MTT-S International Microwave Symposium Digest (MTT), 2013, pp. 1-4

[11]C. I. Trainito, O. Français, et B. Le Pioufle, «Monitoring the permeabilization of a single cell in a microfluidic device, through the estimation of its dielectric properties based on combined dielectrophoresis and electrorotation in situ experiments ", Electrophoresis, vol. 36, n ${ }^{\circ}$ 9-10, pp. 1115-1122, May 2015.

[12]T. García-Sánchez, M. Guitart, J. Rosell-Ferrer, A. M. Gómez-Foix, et R. Bragós, " A new spiral microelectrode assembly for electroporation and impedance measurements of adherent cell monolayers ", Biomed. Microdevices, vol. 16, $\mathrm{n}^{\circ}$ 4, pp. 575-590, Aug. 2014.

[13]T. Chen, D. Dubuc, M. Poupot, J. J. Fournié, et K. Grenier, "Broadband discrimination of living and dead lymphoma cells with a microwave interdigitated capacitor", in 2013 IEEE Topical Conference on Biomedical Wireless Technologies, Networks, and Sensing Systems, 2013, pp. 64-66.

[14]S. Vassanelli et al., "Space and time-resolved gene expression experiments on cultured mammalian cells by a single-cell electroporation microarray ", New Biotechnol., vol. 25, $\mathrm{n}^{\circ}$ 1, pp. 55-67, June 2008

[15]T. Ishibashi, K. Takoh, H. Kaji, T. Abe, et M. Nishizawa, « A porous membrane-based culture substrate for localized in situ electroporation of adherent mammalian cells ", Sens. Actuators B Chem., vol. 128, $\mathrm{n}^{\circ}$ 1, pp. 5-11, Dec. 2007.

[16]A. Adamo, A. Arione, A. Sharei, et K. F. Jensen, «Flow-Through Comb Electroporation Device for Delivery of Macromolecules ", Anal. Chem., vol. 85, nº 3, pp. 1637-1641, Feb. 2013.

[17]C. Dalmay, M. A. De Menorval, O. Français, L. M. Mir, et B. Le Pioufle, «A microfluidic device with removable packaging for the real time visualisation of intracellular effects of nanosecond electrical pulses on adherent cells », Lab. Chip, vol. 12, n 22, pp. 4709, 2012.

[18]C. Merla et al., « A wide-band bio-chip for real-time optical detection of bioelectromagnetic interactions with cells ", Sci. Rep., vol. 8, nº 1 , Dec. 2018.

[19]N. Bao, T. T. Le, J.-X. Cheng, et C. Lu, « Microfluidic electroporation of tumor and blood cells: observation of nucleus expansion and implications on selective analysis and purging of circulating tumor cells », Integr. Biol., vol. 2, no 2-3, pp. 113-120, March 2010.

[20]H. He, D. C. Chang, et Y.-K. Lee, « Micro pulsed radio-frequency electroporation chips ", Bioelectrochemistry Amst. Neth., vol. 68, $\mathrm{n}^{\circ}$ 1, pp. 89-97, Jan. 2006.

[21]H. He, D. C. Chang, et Y.-K. Lee, « Using a micro electroporation chip to determine the optimal physical parameters in the uptake of biomolecules in HeLa cells », Bioelectrochemistry, vol. 70, $\mathrm{n}^{\circ}$ 2, pp. 363-368, May 2007.

[22]Y. Sun, P. T. Vernier, M. Behrend, L. Marcu, et M. A. Gundersen, «Electrode Microchamber for Noninvasive Perturbation of Mammalian Cells With Nanosecond Pulsed Electric Fields », IEEE Trans. Nanobioscience, vol. 4, $\mathrm{n}^{\circ}$ 4, pp. 277-283, Dec. 2005.

[23]E. Ghafar-Zadeh, B. Gholamzadeh, F. Awwad, et M. Sawan, «Onchip electroporation: characterization, modeling and experimental results", Conf. Proc. Annu. Int. Conf. IEEE Eng. Med. Biol. Soc. IEEE Eng. Med. Biol. Soc. Annu. Conf., vol. 2012, pp. 2583-2586, 2012.

[24]Y. Huang, N. S. Sekhon, J. Borninski, N. Chen, et B. Rubinsky, «Instantaneous, quantitative single-cell viability assessment by electrical evaluation of cell membrane integrity with microfabricated devices ", Sens. Actuators Phys., vol. 105, n 1, pp. 31-39, June 2003.

[25]H. Li, X. Ma, X. Du, X. Cheng, et J. C. M. Hwang, " High-frequency continuous-wave electroporation of Jurkat human lymphoma cells », in 2016 IEEE MTT-S International Microwave Symposium (IMS), San Francisco, CA, 2016, pp. 1-4.

[26]A. D. Hargis, J. P. Alarie, et J. M. Ramsey, « Characterization of cell lysis events on a microfluidic device for high-throughput single cell 
analysis », Electrophoresis, vol. 32, n 22, pp. 3172-3179, Nov. 2011.

[27]N. Jokilaakso et al., « Ultra-localized single cell electroporation using silicon nanowires », Lab Chip, vol. 13, n 3, pp. 336-339, Jan. 2013.

[28]M.-S. Hung et Y.-T. Chang, « Single cell lysis and DNA extending using electroporation microfluidic device », BioChip J., vol. 6, n ${ }^{\circ} 1$, pp. 84-90, March 2012.

[29]Y. Huang et B. Rubinsky, « Flow-through micro-electroporation chip for high efficiency single-cell genetic manipulation », Sens. Actuators Phys., vol. 104, no 3, pp. 205-212, May 2003.

[30] Y.-C. Lin, C.-M. Jen, M.-Y. Huang, C.-Y. Wu, et X.-Z. Lin, «Electroporation microchips for continuous gene transfection », Sens. Actuators B Chem., vol. 79, n 2-3, pp. 137-143, Oct. 2001

[31]D. Zhao et al., «A Flow-Through Cell Electroporation Device for Rapidly and Efficiently Transfecting Massive Amounts of Cells in vitro and ex vivo », Sci. Rep., vol. 6, Jan. 2016.

[32]M. Marty et al., «Electrochemotherapy - An easy, highly effective and safe treatment of cutaneous and subcutaneous metastases: Results of ESOPE (European Standard Operating Procedures of Electrochemotherapy) study », Eur. J. Cancer Suppl., vol. 4, n 11, pp. 3-13, Nov. 2006.

[33]L. M. Mir, « Bases and rationale of the electrochemotherapy », Eur. J. Cancer Suppl., vol. 4, n 11, pp. 38-44, Nov. 2006.

[34] Y.-S. Choi, H.-B. Kim, G.-S. Kwon, et J.-K. Park, « On-chip testing device for electrochemotherapeutic effects on human breast cells », Biomed. Microdevices, vol. 11, n 1, pp. 151-159, Feb. 2009.

[35]M. Wu, Y. Xiao, D. Zhao, Z. Liang, et Z. Li, «A microchip for in vitro parameter determination of cancer electrochemotherapy », in 2013 Transducers Eurosensors XXVII: The 17th International Conference on Solid-State Sensors, Actuators and Microsystems (TRANSDUCERS EUROSENSORS XXVII), 2013, pp. 2157-2160.

[36]A. Paganin-Gioanni, E. Bellard, J. M. Escoffre, M. P. Rols, J. Teissié, et M. Golzio, «Direct visualization at the single-cell level of siRNA electrotransfer into cancer cells ", Proc. Natl. Acad. Sci., vol. 108, n 26, pp. 10443-10447, June 2011.

[37] J. Teissie et M. Golzio, «Direct Imaging of siRNA Electrotransfer at the Single-Cell Level », in Electroporation Protocols, Humana Press, New York, NY, 2014, pp. 121-130.

[38]K. A. DeBruin et W. Krassowska, « Modeling Electroporation in a Single Cell. II. Effects of Ionic Concentrations », Biophys. J., vol. 77, n 3 , pp. 1225-1233, Sept. 1999.

[39]A. Tamra, D. Dubuc, M. P. Rols, et K. Grenier, « Microwave Monitoring of Single Cell Monocytes Subjected to Electroporation », IEEE Trans. Microw. Theory Tech., vol. 65, $\mathrm{n}^{\circ}$ 9, pp. 3512-3518, Sept. 2017.

[40]T. Chen, D. Dubuc, M. Poupot, J. J. Fournie, et K. Grenier, « Accurate Nanoliter Liquid Characterization $\mathrm{Up}$ to $40 \mathrm{GHz}$ for Biomedical Applications: Toward Noninvasive Living Cells Monitoring », IEEE Trans. Microw. Theory Tech., vol. 60, $\mathrm{n}^{\circ}$ 12, pp. 4171-4177, Dec. 2012.

[41]S. Kang, K.-H. Kim, et Y.-C. Kim, « A novel electroporation system for efficient molecular delivery into Chlamydomonas reinhardtii with a 3-dimensional microelectrode », Sci. Rep., vol. 5, Nov. 2015.

[42] S. Wang, X. Zhang, W. Wang, et L. J. Lee, « Semi-continuous Flow Electroporation Chip for High Throughput Transfection on Mammalian Cells », Anal. Chem., vol. 81, n 11, pp. 4414-4421, June 2009.

[43]S. C. Bürgel, C. Escobedo, N. Haandbæk, et A. Hierlemann, « Onchip electroporation and impedance spectroscopy of single-cells », Sens. Actuators B Chem., vol. 210, pp. 82-90, Avr. 2015. with the Laboratory of Analysis and Architecture of System, National Scientific Research Center (LAAS-CNRS), Toulouse, France. Her scientific research interests include the use of microwave based dielectric spectroscopy for the study and the characterization of electroporation and electro-transfer of drugs into human cancer cells.

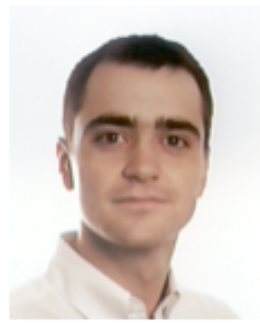

David Dubuc (S'99, M'03) received the Agregation degree from the Ecole Normale Supérieure de Cachan, Paris, France, in 1996, and the M.S. and Ph.D. degrees in electrical engineering from the University of Toulouse, Toulouse, France, in 1997 and 2001, respectively. From 2002 to 2013, he was an Associate Professor with the University of Toulouse, and a Researcher with the Laboratory of Analysis and Architecture of System part of National Scientific Research Center (LAAS-CNRS), Toulouse, France. From 2007 to 2009, he was a Visiting Senior Researcher with the Laboratory of Integrated Micromechatronic Systems (LIMMSCNRS)/Institute of Industrial Science (IIS), University of Tokyo, Tokyo, Japan. Since 2013, he is Professor with the University of Toulouse. His research interests include the development of microwave circuits integrated due to microtechnologies and their application to wireless telecommunication and biology.

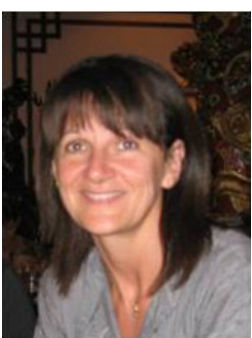

Marie-Pierre Rols received a Master's degree in Biochemistry, a Ph.D. in Cell Biophysics and the Habilitation à Diriger les Recherches from the Paul Sabatier University of Toulouse in 1984, 1989 and 1995, respectively. She is currently Director of Research at the IPBS-CNRS laboratory in Toulouse, vice secretary of the GTRV (Groupe Thématique de recherche sur la Vectorologie), member of the GEM (Groupe d'Etude des Membranes). Her main research interests lie in the fields of membrane electropermeabilization in vitro and in vivo. In the last years, she has focused on the mechanism of DNA electrotransfer. Marie-Pierre Rols is the author of more than 100 articles in peer-reviewed journals. In 1993 she received the Galvani Prize of the Bioelectrochemical Society, in 2006 a joined prize of the Midi-Pyrénées region. She is member of the management committee of the COST TD1104 - European network for development of electroporation-based technologies and treatments (EP4Bio2Med).

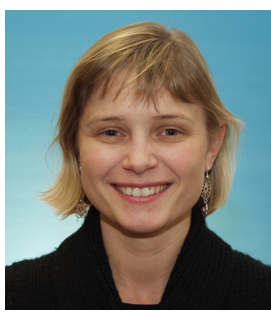

Katia Grenier (S'99, M'03) received her M.S. and $\mathrm{Ph} . \mathrm{D}$. degrees in electrical engineering from the University of Toulouse, Toulouse, France, in 1997 and 2000, respectively. She was engaged in microelectromechanical systems (MEMS) circuits on silicon. She was a Postdoctoral Fellow at Agere Systems (Bell Labs). In 2001, she joined the Laboratory of Analysis and Architecture of System of the National Scientific Research Center (LAAS-CNRS), Toulouse, France. From 2007 to 2009, she was with the Laboratory for Integrated Micromechatronic Systems CNRS (LIMMS-CNRS)/Institute of Industrial Science (IIS), Universtity of Tokyo, Tokyo, Japan, where she was engaged in launching research activities on microwave-based biosensors. Her research interests in LAAS-CNRS are now focused on the development of fluidic-based microsystems, notably for biological and medical applications at the cellular and molecular levels. Dr. Grenier is a member of the IEEE MTT-10 Technical Committee on Biological effect and medical applications of RF and microwave of the IEEE Microwave Theory and Techniques Society.
Amar Tamra received the M.S. degree in molecular diagnostics from the Faculty of Sciences, Lebanese University, Beirut, Lebanon, in 2013. She obtained the Ph.D. degree at the University of Toulouse, Toulouse, France, in 2017. She was performing her research activities 